FORMATION Formation emploi

Revue française de sciences sociales

113 | janvier-mars 2011

La flexicurité à l'aune de l'approche par les capacités

\title{
Sécurisation des parcours et liberté professionnelle : de la « flexicurité » aux capacités
}

Greater career security and professional freedom: from "flexicurity" to capabilities

Laufbahnsicherung und berufliche Freiheit: von der « Flexicurity» zur

"Capability»

Protección de los itinerarios y libertad profesional : de la « flexiguridad » a las capacidades

\section{Pascal Caillaud et Bénédicte Zimmerman}

\section{CpenEdition}

Journals

Édition électronique

URL : http://journals.openedition.org/formationemploi/3230

DOI : 10.4000/formationemploi.3230

ISSN : 2107-0946

Éditeur

La Documentation française

Édition imprimée

Date de publication : 1 mars 2011

Pagination : $33-48$

ISSN : 0759-6340

Référence électronique

Pascal Caillaud et Bénédicte Zimmerman, « Sécurisation des parcours et liberté professionnelle : de la " flexicurité » aux capacités », Formation emploi [En ligne], 113 | janvier-mars 2011, mis en ligne le 10 avril 2013, consulté le 30 octobre 2020. URL : http://journals.openedition.org/formationemploi/3230 DOI : https://doi.org/10.4000/formationemploi.3230

(C) Tous droits réservés 


\section{DOSSIER}

\section{Sécurisation des parcours et liberté professionnelle : de la «flexicurité » aux capacités}

Pascal Caillaud et Bénédicte Zimmerman*

La mise en œuvre française de la flexicurité comme de la formation tout au long de la vie privilégie la formation liée au poste de travail et au maintien dans l'emploi. L'approche par les capacités permet de dessiner un scénario alternatif $d^{\prime}$ articulation entre sécurité et flexibilité, fondé sur la liberté professionnelle.

En 2007, la Commission européenne énonce les principes d'une politique commune de «flexicurité » en tant que « stratégie intégrée visant à améliorer simultanément la flexibilité et la sécurité sur le marché du travail ». L'objectif est de renforcer conjointement la flexibilité économique et la réussite des «changements (les "transitions») dans la vie: entre le système éducatif et le monde du travail, entre les emplois, entre le chômage ou l'inactivité et le travail, entre le travail et la retraite. (...) Il s'agit de faciliter la progression des travailleurs vers de meilleurs emplois, de favoriser la "mobilité ascensionnelle" et le développement optimal des talents » (Commission européenne, 2007, p. 5). La flexicurité ainsi définie n'a donc pas simplement vocation à combiner flexibilité et sécurité. Elle promeut une flexibilité à double sens, favorable à l'entrepreneur et au salarié ; une flexibilité qui consacre non seulement la liberté d'entreprendre, mais encore la liberté de travailler et de se développer professionnellement. À cette fin, la Commission identifie quatre composantes d'une
* Pascal Caillaud, juriste, est chargé de recherche CNRS au Laboratoire "Droit et Changement Social » (UMR CNRS 3128 / université de Nantes), chercheur à la Maison des Sciences de l'Homme Ange Guépin de Nantes et directeur du Centre associé au Céreq des Pays de la Loire. II est notamment l'auteur, avec Guy Brucy, Emmanuel Quenson et Lucie Tanguy, de Former pour réformer, Retour sur la formation permanente (1959-2004), Paris, La Découverte, 2007, et Le diplôme, Paris, LGDJ, à paraître en 2011.

Bénédicte Zimmermann, sociologue, est directrice d'études à l'École des hautes études en sciences sociales à Paris et membre du Centre de recherche interdisciplinaire sur l'Allemagne (CNRS/EHESS). Elle a notamment publié : La constitution du chômage en Allemagne, Paris, Ed. de la MSH, 2001 et Ce que travailler veut dire. Une sociologie des capacités et des parcours professionnels, Paris, Economica, 2010. 
politique commune de flexicurité : 1. la "souplesse et la sécurisation des dispositions contractuelles » 2. « des stratégies globales d'apprentissage tout au long de la vie» 3. "des politiques actives du marché du travail efficaces » 4. "des systèmes de sécurité sociale modernes » (Idem, pp. 5-6).

Ces composantes se concentrent sur des dimensions juridiques et institutionnelles - l'emploi, la formation, les politiques du marché du travail et de protection sociale -, mais placent au second rang l'articulation de la flexibilité et de la sécurité dans l'activité même de travail. Notre hypothèse est qu'une telle architecture ne rompt pas avec le schéma dual qui situe la flexibilité du côté de l'entreprise, dans la sphère économique, et la sécurité du côté du travailleur, dans la sphère sociale ; schéma en vertu duquel la sécurité constitue un amortisseur externe des conséquences sociales de la flexibilité des entreprises. Asymétrique, une telle conception réduit l'objectif d'une flexibilité à double sens à un vœu pieux, tout en dédouanant les entreprises de leurs responsabilités en matière de sécurité et de développement professionnel des salariés.

Nos recherches montrent au contraire que l'entreprise est un maillon essentiel pour articuler flexibilité et sécurité, dès lors que l'on conçoit ces deux dimensions orientées à la fois vers l'entreprise et les salariés. La sécurité des salariés ne se joue pas seulement dans l'emploi et l'aménagement de transitions sur le marché du travail ; elle se forge aussi dans le travail à proprement parler et les possibilités de développement professionnel qu'offre ce dernier. La précarité n'est pas seulement liée à l'emploi et sa forme juridique au type de contrat notamment; elle découle aussi des conditions concrètes de travail qui peuvent être source de développement ou au contraire de vulnérabilité professionnelle. La flexibilité externe est la principale cible des politiques européennes ; dès lors, il nous semble indispensable de considérer également la flexibilité interne à l'entreprise et de mieux thématiser les liens entre le parcours des salariés à l'intérieur de l'entreprise et leur devenir sur le marché externe.

L'objectif de notre propos est double. Il s'agit, dans un premier temps, d'analyser la transposition, dans le droit français, du deuxième pilier de la flexicurité relatif à l'apprentissage tout au long de la vie. La législation française préfère à flexicurity la notion de " sécurisation des parcours », à lifelong learning celle de «formation professionnelle »; elle introduit par ailleurs l'idée de « liberté contractuelle». Après l'analyse de cette traduction sémantique et juridique, nous confronterons, dans un second temps, les droits formels qui en résultent à leur mise en œuvre par les entreprises et leurs conséquences pour les salariés. Nous mobiliserons deux enquêtes qualitatives sur le développement professionnel des salariés, menées en 2005 et 2009 auprès de treize entreprises françaises ${ }^{1}$ Ces enquêtes montrent que les droits à la formation contribuent à la sécurité et à la mobilité du travailleur à condition que leur mise en œuvre ouvre un espace de liberté professionnelle que nous caractériserons par l'existence avérée de capacités de développement professionnel, au sens de l'approche par les capacités initiée par A. Sen.

\section{DE LA FLEXICURITÉ À LA SÉCURISATION DES PARCOURS}

Les réformes appelées à être mises en place dans le cadre de la flexicurité portent aussi bien sur les structures générales des systèmes de formation que sur les entreprises ou les droits des personnes. Or, selon les articles 149 et 150 du Traité instituant la Communauté européenne, ces champs relèvent exclusivement des compétences juridiques des États. Par conséquent, la flexicurité et la formation tout au long de la vie relèvent, au niveau européen, de la « méthode ouverte de coordination » (MOC). Celle-ci ne produit pas des actes juridiques communautaires contraignants, mais une « littérature grise » (communications, recommandations, avis et résolutions) qui vise la convergence des politiques nationales par le biais d'obligations de résultats assumées par les États (Pochet, 2004). Une double transposition des textes européens en droit national est donc nécessaire : transposition des concepts, d'une part, transposition des dispositifs promus par les politiques communautaires, d'autre part.

\footnotetext{
${ }^{1}$ Enquêtes successivement menées avec Delphine Corteel et Dilip Subramanian dans le cadre des projets européens «Eurocap : Dialogue Social, Emploi et Territoire, pour une politique européenne des capacités » (http://www.idhe.ens-cachan.fr/Eurocap/) et «Capright: Resources, Rights and Capabilities. In Search of Social Foundation for Europe » (www.capright.eu)
} 
Une transposition aménagée des concepts dans le droit français

Lifelong learning comme flexicurity sont deux anglicismes, apparus, pour le premier, dans les sphères d'experts internationaux (Hasan, 1997), pour le second, dans les politiques publiques portant sur le marché du travail, aux Pays-Bas, puis au Danemark (Gaudu, 2008). Progressivement intégrées dans les politiques communautaires, ils font l'objet d'adaptations tant linguistiques que conceptuelles dans leur mise en œuvre par le droit français.

\section{Flexicurity : une terminologie écartée du droit}

Ce néologisme est né de la contraction des deux termes de flexibility et de security. La notion de sécurité est bien établie dans le langage du droit, notamment français : la santé et la sécurité physique des travailleurs - sécurité dans le travail - est au principe des premières règles du droit du travail, qu'il s'agisse des droits nationaux ou de l'embryonnaire droit social européen ${ }^{2}$. Son périmètre fut progressivement étendu à la « sécurité par le travail » (Supiot, 2002) qui pose la question de la subsistance du travailleur et de sa sécurité économique : droit au salaire, à la continuité des revenus face à certains événements (chômage, maladie, âge...). Cette deuxième acception de la sécurité du travailleur (capacité physique de travailler (Deakin et Supiot, 2010)) est visée dans le concept de flexicurité, mais de manière restrictive. En effet, au sens large, la sécurité par le travail a conduit au développement de législations qui stabilisent la relation de travail (congés, suspension, protection et maintien du contrat). Or telle n'est pas la finalité de la sécurité dans la flexicurité qui vise la réussite des transitions tout en « aidant les travailleurs à s'adapter et accepter le changement» (Commission européenne, 2007).

Le terme de flexibilité a longtemps été frappé d'irrecevabilité politique en France. En 1997, Martine Aubry, alors ministre du Travail, considère qu'il s'agit d'un vilain mot qu'il vaut mieux remplacer par « souplesse »; cette option a prévalu dans la version

\footnotetext{
2 " L'employeur est obligé d'assurer la santé et la sécurité des travailleurs dans tous les aspects liés au travail », Directive 89/391/ CEE du 12 juin 1989 relative à l'amélioration de la sécurité des travailleurs et de la santé au travail.
}

française des résolutions pour l'emploi du Sommet européen du Luxembourg de novembre 1997. De même, Jacques Chirac, une fois Président de la République, fait savoir que «la connotation antisociale du mot flexibilité ne (lui) plaît guère » ${ }^{3}$. Face à ces réticences, plutôt que de flexibilité, le droit français use du concept de liberté contractuelle ou professionnelle (Gaudu, 2008), aussi bien pour l'employeur que pour le salarié. De même, en lieu et place du concept binaire de flexicuritét, il préfère accentuer la composante de sécurité à travers la « sécurisation des parcours professionnels », dans un rapprochement sémantique avec la Sécurité Sociale, perçue comme une conquête sociale.

La notion de parcours renvoie de manière euphémisée, dans la version française, à l'idée de mobilité et de flexibilité. Elle signifie, dans son sens le moins restrictif, une continuité de l'expérience et des droits par-delà la diversité des statuts et l'instauration de passerelles entre les différentes situations de la vie active : entre différents emplois, des périodes d'activité et d'inactivité, de formation ou de chômage. Son introduction dans le langage juridique n'est pas l'apanage du droit de la formation professionnelle. On trouve le terme, dès le début des années 80 , dans les textes relatifs à l'éducation et à l'insertion professionnelle $^{5}$. Cette législation porte essentiellement sur la mise en œuvre de politiques publiques qui, sans créer de droits subjectifs ${ }^{6}$, n'en témoigne pas moins d'une volonté du législateur d'intégrer, dans un statut juridique cohérent, un certain nombre d'actes et de dispositifs juridiques interdépendants, convergeant vers un même but. La difficulté de traduire concrètement la notion de parcours en termes de droit subjectif de la personne, que l'on qualifie aujourd'hui de droit opposable, explique sans doute, sinon la réticence initiale du moins le faible recours du législateur à un mot pourtant fortement répandu dans le discours public.

\footnotetext{
3 Déclaration télévisée du 12 décembre 1996. Rappelée par Le Monde, Cahier Initiatives, 6 janv. 1999.

4 Présentée comme étape "essentielle dans l'instauration d'une flexisécurité » française (www.gouvernement.fr), la loi du 25 juin 2008 ne consacre pas cette expression pour retenir celle de « modernisation du marché du travail ».

5 Article L. 5312-1 du Code du travail.

${ }^{6}$ On peut définir le droit subjectif comme «un droit qui suppose un tiers à qui il est opposable: c'est le droit de faire valoir une prérogative à l'encontre d'autrui » (Gutmann, 2003, p. 530).
} 
Antérieurement à toute consécration conventionnelle ou législative, la notion de parcours professionnel fut utilisée par la Cour de cassation dans un contentieux relatif aux dérogations au principe «travail égal, salaire égal ». Aux yeux du juge français, les « parcours professionnels spécifiques » des salariés accomplissant pourtant la même prestation de travail, peuvent néanmoins justifier qu'ils ne perçoivent pas la même rémunération ${ }^{7}$. Les partenaires sociaux vont finalement porter cette notion dans le langage juridique (Luttringer et Willems, 2007), à travers la promotion du «parcours de formation » (préambule de l'Accord national interprofessionnel [ANI] du 5 décembre 2003), puis finalement du «parcours professionnel $»^{8}$. La transposition de ces accords a eu pour effet d'intégrer la notion au niveau législatif. Cependant, le caractère hétérogène des parcours qui mêlent aussi bien formation professionnelle, gestion prévisionnelle de l'emploi et des compétences (GPEC) que mécanismes de retour à l'emploi, ne permet pas de concevoir le « parcours professionnel» comme un dispositif unique et commun à tous les statuts d'actifs (salariés, agents publics, demandeurs d'emplois), mais plutôt comme un encadrement juridique de dispositifs parcellaires souvent complexes (Vatinet, 2007).

\section{Lifelong learning : le prisme réducteur de la formation professionnelle tout au long de la vie}

Selon les textes européens, le " lifelong learning" doit couvrir la vie entière, depuis la période préscolaire jusqu'à l'âge de la retraite, incluant l'éventail complet de l'éducation et de la formation formelle, non formelle et informelle ${ }^{10} \gg$ (Commission euro-

\footnotetext{
7 Cass. Soc, 3 mai 2006, 03-42920.

${ }^{8}$ De l'ANI du $1^{\text {er }}$ mars 2004 relatif à la mixité et l'égalité professionnelle à l'ANI du 9 janvier 2009 sur le développement de la formation tout au long de la vie professionnelle, la professionnalisation et la sécurisation des parcours professionnels.

9 « Éducation et formation professionnelle tout au long de la vie » constitue la traduction officielle de lifelong learning (Commission européenne, 2001), la « formation professionnelle tout au long de la vie » (Sixième partie du Code du travail) se traduisant par « lifelong vocational training ».

${ }_{10}$ Selon la terminologie communautaire, «l'apprentissage formel est généralement dispensé par des établissements d'enseignement ou de formation, avec des objectifs d'apprentissage structurés, une durée d'apprentissage et un soutien fourni. Il est intentionnel de la part de l'apprenant et entraîne une certification; l'apprentissage non formel ne relève pas d'un établissement d'enseignement ou de formation et ne mène généralement à aucune certification. Il est toutefois intentionnel de la part de l'apprenant et présente
}

péenne, 2001). Mais l'absence de caractère obligatoire de cette définition donne lieu à des mises en œuvre nationales contrastées. En France, en 2003, les organisations patronales et syndicales signent l'ANI « relatif à l'accès des salariés à la formation tout au long de la vie professionnelle ». La formation y est conçue de manière restrictive en référence à un type de public (les salariés) et une période au cours de laquelle ce public sera amené à se former (toute la vie professionnelle); et ce au détriment d'une conception plus extensive qui dépasserait les frontières liées au statut des personnes et aux âges de la vie. C'est d'ailleurs dans la formulation révisée de « formation professionnelle tout au long de la vie » que la notion intègre l'ordre juridique français avec la loi de 2004, au motif qu'elle " est celle utilisée par les textes européens et doit être à ce titre maintenue $»^{11}$, quand bien même le contenu de la loi et son spectre d'application, qui eux n'ont pas été révisés, ne satisfont pas à l'ambition de la formulation européenne.

En effet, telle qu'elle est définie par le législateur ${ }^{12}$, la «formation professionnelle tout au long de la vie » s'inscrit dans un objectif uniquement professionnel : actualiser des connaissances et des compétences favorisant l'évolution professionnelle de chaque personne et, depuis 2009, progresser d'au moins un niveau de qualification au cours de sa vie professionnelle ${ }^{13}$. Beaucoup plus large, l'acception européenne du lifelong learning doit permettre de poursuivre une formation dans « une perspective personnelle, physique, sociale ou en vue d'un emploi» (Commission européenne, 2001).

des délais, un soutien et des objectifs structurés; l'apprentissage informel résulte d'activités quotidiennes liées au travail, à la vie de famille ou aux loisirs. Non structuré, il n'entraîne généralement pas de certification. Dans la plupart des cas, il n'est pas intentionnel de la part de l'apprenant ». (Conclusions du Conseil sur des principes européens communs pour l'identification et la validation de l'éducation et de la formation non formelles et informelles, mai 2004).

11 Réponse du ministre du Travail français à la demande d'explications de parlementaires, Journal Officiel, débats Assemblée nationale $1^{\text {re }}$ séance du 12 décembre 2003, p. 12307.

12 Loi du 24 novembre 2009 relative à l'orientation et à la formation professionnelle tout au long de la vie.

13 Article L. 6111-1 du Code du travail. Rappelons que la sixième partie du Code du travail, relative à « la formation professionnelle tout au long de la vie », ne porte que sur l'apprentissage, la formation continue, la VAE (validation des acquis de l'expérience) et le rôle des institutions dans ces domaines. Ce faisant, la formation professionnelle initiale reste du domaine du Code de l'éducation, alors qu'elle fait partie intégrante du concept de formation tout au long de la vie. 


\section{Formation, sécurisation des parcours et liberté professionnelle}

Si la mise en œuvre française de la flexicurité ${ }^{14}$ accentue la «sécurisation des parcours professionnels », elle sollicite aussi le concept juridique de liberté professionnelle, entendu au sens de liberté contractuelle, c'est-à-dire du renforcement de la liberté des parties au contrat de travail (Gaudu, 2008), notamment d'aménager les modalités de départ et d'exécution de la formation. Mais cette liberté ne s'oriente pas vers la création d'un droit individuel à la formation qualifiante qui constituerait pourtant un fondement décisif de la liberté professionnelle.

\section{Formation et sécurisation des parcours professionnels}

Invoquée pour répondre à "l'insécurité de l'emploi, l'insécurité des parcours professionnels, l'insécurité des modes de ruptures du contrat de travail, l'insécurité du droit, tout simplement » (Favennec-Héry, 2007), l'idée de sécurisation reste floue. Initialement sollicitée pour réduire les risques liés aux transitions professionnelles, en conférant aux salariés ou aux demandeurs d'emploi des droits d'accès à des dispositifs ${ }^{15}$ (Gaudu, 2008), la " sécurisation des parcours professionnels » s'est plus récemment orientée vers l'instauration d'un mécanisme de collecte de fonds. Ainsi, l'ANI du 9 janvier 2009 a créé le fonds paritaire de sécurisation des parcours professionnels (FPSPP). $\mathrm{Ce}$ fonds a pour objet d'apporter des ressources supplémentaires pour la formation de publics cibles, par des mécanismes de contractualisation et de cofinancements. Concrètement, il s'agit d'abonder et de renforcer le financement de dispositifs déjà existants de qualification et de requalification.

\footnotetext{
${ }^{14}$ La commission Spidla a eu une vision assez restrictive de la traduction française de la flexicurité, puisque seuls apparaissent dans son rapport le DIF (droit individuel à la formation) et la GPEC (gestion prévisionnelle des emplois et des compétences). Rapport de la Mission pour la flexicurité "Mise en ouvre des principes communs de flexicurité dans le cadre du cycle 2008-2010 de la Stratégie de Lisbonne », SOC 776ECOFI_606, décembre 2008.

${ }^{15}$ La sécurisation des parcours professionnels a été consacrée par le droit français, en 2006, pour regrouper des dispositions éparses relatives à la mise à disposition, au congé de mobilité ou au contrat de transition professionnelle. Loi $\mathrm{n}^{\circ} 2006-1770$ du 30 décembre 2006 pour le développement de la participation et de l'actionnariat salarié et portant diverses dispositions d'ordre économique et
} social.
L'introduction de la sécurisation des parcours dans le droit français n'a entrâné ni droit personnel nouveau, ni dispositif supplémentaire d'accès à la formation. Tout au plus, les partenaires sociaux et le législateur de 2009, prenant conscience de l'insuffisance de la transférabilité du DIF (droit individuel à la formation) ont-ils introduit sa portabilité afin d'offrir une possibilité au salarié d'en bénéficier après la rupture de son contrat, soit auprès de son nouvel employeur, soit en tant que demandeur d'emploi. De prime abord, ce mécanisme pourrait être analysé comme l'ébauche d'un dispositif individuel de sécurisation, voire d'un droit personnel lié au travailleur lui-même, plus qu'à son statut (Maggi-Germain et Caillaud 2007). Mais la façon dont la portabilité du DIF a été envisagée consacre une procédure contractuelle plus qu'un réel droit individuel. Sa mise en œuvre ne repose pas sur un droit discrétionnaire de son titulaire mais nécessite l'accord impératif du nouvel employeur ou, dans le cas d'un demandeur d'emploi, l'avis du référent chargé de l'accompagnement ${ }^{16}$.

Cela signifie-t-il que le droit de la formation professionnelle ne peut donner consistance à la notion de sécurisation autrement que par l'apport de financements? La jurisprudence permet de nuancer le propos. Depuis 1992, se fondant sur l'article 1134 du Code civil selon lequel «les conventions doivent être exécutées de bonne foi » par les parties, la Cour de Cassation fait peser sur l'employeur un devoir d'assurer l'adaptation des salariés à l'évolution de leurs emplois ${ }^{17}$. Cette obligation, transposée dans le Code du travail par la loi Aubry II ${ }^{18}$, a été étendue par la loi du 4 mai 2004. Celle-ci impose à l'employeur " d'assurer l'adaptation des salariés à leur poste de travail », mais également de "veiller au maintien de leur capacité à occuper un emploi, au regard notamment de l'évolution des emplois, des technologies et des organisations $»^{19}$. Peut-on ici parler de sécurisation des parcours professionnels ? La réponse nous semble être positive puisqu'il s'agit de sécuriser la relation de travail et d'en éviter la rupture (Maggi-Germain, 2008), en considérant que l'absence de formation,

\footnotetext{
16 Article L.6323-17 du Code du travail.

17 Cass. Soc. 25 février 1992, n 89-41634.

${ }^{18}$ Loi n $^{\circ}$ 2000-37 du 19 janvier 2000 relative à la réduction négociée du temps de travail.

19 Article L. 6321-1 du Code du travail.
} 
entendue au sens large, constitue un préjudice spécifique pour lequel le salarié doit être indemnisée ${ }^{20}$.

\section{Formation et liberté contractuelle}

À partir de la loi de $1971^{21}$, le départ en formation du salarié s'inscrit dans le cadre du contrat de travail. Lorsque le salarié est demandeur de la formation et ne se trouve pas en mesure d'effectuer sa prestation de travail, le contrat de travail est suspendu dans le cadre du congé individuel de formation (CIF - congé individuel de formation). À l'inverse, lorsque la formation est à l'initiative de l'employeur, elle est assimilée à du travail effectif, le départ en formation étant alors un ordre. Depuis le début des années 2000, cette conception a progressivement été reconsidérée par des réformes qui renforcent la liberté du salarié et de l'employeur de conclure des accords sur les modalités du départ, de la prise en charge et de la reconnaissance de la formation. Ainsi, le Plan de formation et le DIF prévoient dorénavant la possibilité que la formation s'exécute, en partie (Plan de formation) ou en totalité (DIF), hors du temps de travail effectif du salarié, dès lors que celui-ci exprime son accord par écrit.

Pour justifier l'introduction de ces accords spécifiques dans le contrat de travail, le législateur invoque le bénéfice professionnel et financier que le salarié peut retirer de la formation. D'abord, ces accords visent des actions de développement de compétences (Plan) ou d'actions de promotion, d'acquisition, d'entretien ou de perfectionnement des connaissances (DIF), c'est-àdire de formations bénéficiant au salarié, qu'il pourra faire valoir dans le cadre d'une mobilité. Ensuite, ce temps personnel est désormais indemnisé par le versement d'une allocation de formation qui, si elle n'est pas du même niveau que la rémunération habituelle, n'en constitue pas moins une évolution par rapport à la législation antérieure. Enfin, l'employeur doit prendre des engagements sur la reconnaissance de la formation en accordant au salarié une priorité d'accès aux postes correspondant aux connaissances acquises.

${ }^{20}$ Cass. Soc. 23 octobre 2007, n 06-40950. Cette jurisprudence s'applique également aux salariés auxquels n'avait été proposée, pendant toute la durée dans l'entreprise, aucune formation pour combattre l'illettrisme dû à leur origine étrangère. Cass. Soc. 2 mars 2010, n 09-40914 09-40915 09-40916 09-40917.

${ }^{21}$ Loi $\mathrm{n}^{\circ} 71-575 \mathrm{du} 16$ juillet 1971 portant organisation de la formation professionnelle continue dans le cadre de l'éducation permanente.
On peut toutefois douter de l'équilibre de ce type d'accord. D'une part, il s'inscrit dans une relation de subordination. Dans quelle mesure le salarié est-il en situation d'égalité juridique avec son co-contractant, quand il accepte d'investir de son temps personnel ? Il est difficile de vérifier si son accord ne résulte pas d'une contrainte de fait. L'engagement patronal sur la reconnaissance de la formation doit lui aussi être relativisé. En effet, la priorité d'accès à un poste, limitée à un an, repose sur la volonté de l'employeur, seul juge de l'aptitude d'un salarié à satisfaire les exigences d'un emploi. D'autre part, on peut contester la conception selon laquelle la formation du plan est désormais un avantage quasi-contractuel pour le salarié, alors qu'elle relevait traditionnellement de la responsabilité de l'employeur.

\section{L'introuvable droit individuel à la formation qualifiante}

La définition de l'éducation et de la formation tout au long de la vie par le Conseil européen ${ }^{22}$ s'appuie expressément sur l'article 14 de la Charte des droits fondamentaux de l'Union européenne ${ }^{23}$, qui met l'accent sur la personne et ses droits. Mais comment concevoir un droit à la formation réellement lié à la personne du travailleur, indépendamment de son statut (Maggi-Germain et Caillaud, 2007) ? On peut se référer à la catégorisation des droits sociaux, opérée par le rapport Supiot, à travers la notion « d'état professionnel des personnes »; état appelé à intégrer «les exigences de la liberté du travail, entendue comme liberté concrète, et faciliter le passage d'un type de travail à un autre » (Supiot, 1999). Le concept juridique de capacité pourrait dorénavant être mobilisé en lieu et place de celui d'état professionnel ${ }^{24}$; tous deux recouvrent différents types de droits sociaux : ceux propres au travail salarié, ceux du droit commun de l'activité professionnelle et ceux fondés sur le travail

${ }^{22}$ Résolution du Conseil du 27 juin 2002 sur l'éducation et la formation tout au long de la vie. Journal officiel n C $163 \mathrm{du}$ 09/07/2002, p. 1-3.

23 « Toute personne a droit à l'éducation ainsi qu'à l'accès à la formation professionnelle et continue ».

${ }^{24}$ Selon Alain Supiot, le concept juridique de capacité, issu du droit romain, a sens aussi bien en pays de Common law (où est née l'approche par les capacités) que d'Europe continentale (où a été développée la notion d'état professionnel). La capacité, en droit du travail, possède trois facettes : capacité physique, capacité professionnelle et capacité collective (Deakin et Supiot, 2009). 
non professionnel ${ }^{25}$. La création d'un droit individuel à la formation est susceptible de relever de ces trois types. Il pourra s'agir d'un droit subjectif, directement opposable par un salarié envers son employeur ou d'un droit de tirage social ${ }^{26}$ destiné « à jouer entre le travailleur et la société sur le mode des autres droits sociaux » (Supiot, 1997).

Telle est l'idée de la formation qualifiante différée (ou initiale différée) retenue par les partenaires sociaux en 2003 et 2009, mais rejetée par le législateur. Ce dispositif prévoit que les salariés ayant arrêté leur formation initiale avant ou au terme du premier cycle de l'enseignement supérieur, et en priorité ceux qui n'ont pas obtenu de qualification professionnelle reconnue et qui souhaitent reprendre des études, devraient avoir accès à une ou des formations qualifiantes ou diplômantes, dans le cadre du CIF notamment. Pour les partenaires sociaux, la mise en œuvre de ce droit suppose que les salariés concernés puissent bénéficier d'un abondement financier des pouvoirs publics correspondant au coût moyen d'une année de formation. Au cours des débats parlementaires de la loi de 2009, l'opportunité de la création de ce dispositif fut abordée. À l'amendement proposant son instauration, le secrétaire d'État émit un avis défavorable, rejetant l'option d'un droit opposable, c'est-à-dire d'un droit subjectif. Résultat, la formation différée se limite, dans la loi, à la possibilité offerte à tout apprenti dont la formation n'a pas été sanctionnée par une certification de bénéficier d'une VAE (validation des acquis de l'expérience) pour l'obtention d'un certificat de qualification professionnelle ${ }^{27}$.

Par-delà l'enjeu d'un droit opposable, ce débat entre partenaires sociaux et pouvoirs publics sur l'opportunité de créer un droit à la formation qualifiante soulève la question de la responsabilité et de la prise en charge financière de l'absence de qualification à la

\footnotetext{
25 Selon le rapport Supiot, la quatrième catégorie de droits sociaux, « universels », c'est-à-dire garantis à tous, indépendamment de tout travail, doit faire l'objet de législations spécifiques (Supiot, 1999).

${ }^{26}$ " Droit de tirage car leur réalisation dépend d'une double condition : la constitution d'une provision suffisante et la décision de leur titulaire d'user de cette provision. Droit de tirages sociaux puisqu'ils sont sociaux aussi bien dans leur mode de constitution (abondement différé de la provision) que dans leurs objectifs (utilité sociale) » (Supiot, 1997).

27 Article 34 de la loi du 24 novembre 2009. C'est du moins ainsi que l'exprime le rapporteur du projet à l'Assemblée nationale, séance du 16 juillet 2009, Journal officiel débats, p. 6486.
}

sortie du système scolaire. Pour les représentants des employeurs et des salariés, ce poids doit être assumé par les pouvoirs publics, compétents en matière de formation initiale. Ces derniers, quant à eux, se défaussent sur les partenaires sociaux, compétents en matière de formation professionnelle, selon une partition qui illustre le préjudiciable gouffre français entre formation initiale et formation continue. Ainsi, restreinte à un contenu uniquement professionnel, la formation continue « à la française » ne signifiet-elle pas un droit réel de formation tout au long de la vie pour les salariés. Elle s'entend, à titre principal, comme formation d'adaptation professionnelle, au détriment de la formation qualifiante, privant dans les faits la majeure partie des recalés du système de formation initiale de toute deuxième chance.

L'analyse de la mise en œuvre juridique française des concepts de flexicurity et de lifelong learning révèle une orientation des partenaires sociaux comme du législateur vers des mécanismes de nature contractuelle, au détriment de l'institution d'un réel droit à la formation attaché à la personne. Il nous parait dès lors nécessaire de confronter ces droits formels avec leur mise en œuvre par les entreprises et leurs conséquences pour les salariés. Pour ce faire, deux enquêtes qualitatives sur le développement professionnel des salariés seront mobilisées ${ }^{28}$.

\section{DE LA FORMATION AU DÉVELOPPEMENT PROFESSIONNEL}

L'enquête qualitative permet d'identifier trois types d'entreprises considérées du point de vue de leur politique de formation : des organisations d'actualisation des compétences, des organisations apprenantes et des organisations capacitantes ${ }^{29}$. Dans les premières, l'objectif est d'assurer l'adaptation des salariés aux

\footnotetext{
${ }^{28}$ Enquêtes menées en 2005 et 2009 auprès de treize entreprises françaises.

${ }^{29}$ Cette typologie, centrée sur les politiques de formation, leur mise en œuvre et leurs effets, croise trois dimensions constitutives de l'entreprise : le type de management, l'organisation du travail et la politique des ressources humaines; notre hypothèse étant que la politique de formation, loin d'être du seul ressort de la dernière, s'actualise dans l'interaction entre ces trois dimensions. Chaque type en reflète une configuration spécifique. Pour une présentation détaillée, $c f$. Subramanian et Zimmermann, à paraître en 2011.
} 
exigences, éventuellement changeantes, du poste de travail, sans considération de transférabilité des compétences ou de développement professionnel. Dans les organisations apprenantes, l'activité de travail est elle-même source de connaissances nouvelles et les savoirs acquis sont dotés d'un important degré de généralité et de transférabilité. Cependant, la politique de formation y est exclusivement pilotée par un souci de développement global de l'organisation, au détriment des projets de développement individuels. Enfin, les organisations capacitantes peuvent être définies comme des organisations apprenantes qui, en outre, encouragent et soutiennent les projets individuels de développement. Nous centrerons ici notre propos sur le troisième type, l'organisation capacitante. Il s'agit d'analyser les conditions dans lesquelles, à l'encontre des pratiques françaises dominantes, la formation peut être source de sécurisation des parcours.

Du concept de capacité emprunté à Amartya Sen (2003), nous retiendrons trois traits principaux : la possibilité de choisir entre différentes options, le pouvoir de réaliser l'option choisie et, enfin, l'existence de supports collectifs soutenant le pouvoir individuel d'agir ${ }^{30}$. Nous mènerons l'analyse à partir du cas Bigtrucks, organisation capacitante la plus favorable, dans notre échantillon, à la capacité à se former des salariés ${ }^{31}$. Bigtrucks est un assembleur de poids lourds de plus de quatre cent salariés. L'organisation du travail à la chaîne y est taylorienne, mais revue par des dispositifs de management participatif. La grande majorité des salariés sont des ouvriers qui travaillent sur une ligne d'assemblage non robotisée. La pyramide des fonctions, extrêmement tassée, offre peu d'opportunités pour sortir, via le marché interne, du statut d'opérateur. Pourtant, les salariés disent s'y sentir bien. Le développement professionnel y est avéré, même s'il ne se traduit pas forcément par une évolution de carrière mais passe par des dispositifs participatifs et une politique de formation qui

\footnotetext{
${ }^{30}$ Pour une transposition de la problématique des capacités au monde du travail, $c f$. De Munck et Zimmermann, 2008.

31 Pour une présentation de la méthodologie d'enquête fondée sur l'observation, l'étude de documents et des entretiens semi-directifs avec des salariés de tous niveaux hiérarchiques et des délégués
} syndicaux, $c f$. Corteel et Zimmermann, 2007. dotent les salariés d'une réputation de « qualité » sur le marché externe ${ }^{32}$.

Alors que les débats français sur la flexicurité mettent l'accent sur la sécurisation des parcours, le cas Bigtrucks révèle que la liberté professionnelle constitue une composante importante de la sécurité. La conceptualisation de la liberté en termes de capacité permet d'en préciser les enjeux et de montrer en quoi la sécurisation des parcours a d'autant plus de chance d'être effective qu'elle est associée à l'exercice d'une liberté réelle de mobilité professionnelle. La formation en est un facteur important, mais pas n'importe quelle formation, encore faut-il qu'elle participe au développement professionnel du salarié. Nous dirons d'une formation qui contribue à la fois à la sécurité et à la liberté professionnelle du salarié qu'elle est capacitante en ce qu'elle est source de développement et de pouvoir d'agir ${ }^{33}$.

\section{Les limites de la « logique de compétences»}

Les directives européennes pour l'emploi ont fait de l'employabilité une pièce maîtresse des réformes de la protection sociale. L'employabilité substitue une logique préventive dite « active » à la logique collective dite «passive» des assurances sociales. Elle fait des compétences son maître mot. En théorie, le développement des compétences ${ }^{34}$ vise à «donner aux individus les compétences qui leur permettent de progresser dans leur vie professionnelle et de les aider à trouver un nouvel emploi. » (Commission européenne, 2007, p. 5). Dans les faits, l'enquête montre cependant que la logique compétences responsabilise le salarié en matière de mise à jour et de développement de ses compétences, sans que les conditions d'exercice d'une telle responsabilité soient forcément réunies dans l'entreprise (Corteel et Zimmermann, 2007). Susceptible de contribuer au développement professionnel de certains, cette

\footnotetext{
${ }^{32}$ Pour une étude approfondie du cas Bigtrucks, $c f$. Zimmermann, 2010 .

${ }^{33}$ Une enquête du Céreq montre que sur un échantillon de 4800 entreprises, seules 10,5\% favorisent la capacité à se former de leurs salariés (Lambert et al., 2009).

34 Notion explicitement visée en France, mais sans être définie, par l'article L. 6321-6 du Code du travail, relatif au plan de formation.
} 
logique est productrice d'insécurité pour ceux qui sont renvoyés à l'obsolescence de leurs compétences.

Dans l'entreprise, c'est le supérieur hiérarchique qui décide de qui se formera, à quoi et comment. Le DIF n'a guère changé la donne en la matière, contrairement aux promesses dont il pouvait être porteur. Parce que la compétence s'exerce et s'apprécie en situation de travail, l'entreprise revendique en outre le monopole de son identification et de son évaluation. Or ce monopole est le plus souvent exercé en dehors de toute information et transparence sur la procédure, de toute règle claire de validation et de transférabilité d'une entreprise à une autre. La logique de compétences met la personne au service d'objectifs d'efficacité économique et de performance. Elle vise à accroître le rendement du « capital humain » dans l'entreprise. Elle retient de l'humain ce qui peut directement servir cette visée : la polyvalence, l'adaptabilité, la prise d'initiative et de responsabilité. Être compétent, c'est savoir réagir de la manière qui convient, être capable de faire face à une situation nouvelle ou inhabituelle. La logique de compétences apporte ainsi une réponse à l'impératif économique de réactivité et d'ajustement en juste à temps. Cependant, elle fait peu de place au développement professionnel, à la prise en compte de la dynamique temporelle et sociale des parcours individuels, des préférences et des choix personnels.

Aborder la formation dans l'entreprise au travers des capacités plutôt que des compétences invitent à sortir de cette réduction de l'humain à un capital destiné à fructifier selon la loi du marché. Il s'agit de prendre en compte l'ensemble des dimensions de l'homme au travail, sa performance, ses compétences, mais aussi ses besoins propres, de reconnaissance, de justice, de respect et de sécurité. Les capacités ouvrent alors une voie pour penser conjointement le développement économique de l'entreprise et le développement des personnes au travail. Elles prennent en compte aussi bien ce qu'une personne est capable de faire - ses compétences - que les possibilités réelles de développer et de mettre en œuvre ces compétences - à savoir les opportunités et les moyens d'agir constitutifs des capacités. Si la compétence désigne l'exercice de la responsabilité en situation de travail, la capacité désigne en outre les moyens d'exercer cette responsabilité. Or il n'est point d'exercice de responsabilité sans latitude de choix entre au moins deux options possibles. C'est la raison pour laquelle Sen fait de la liberté de choisir une dimension décisive des capacités et l'érige en objet de responsabilité sociale (Sen, 2003). Le pouvoir d'agir et le potentiel de développement sont les deux autres dimensions qui distinguent les capacités des compétences. Elles intègrent des possibilités de formation qualifiante pour ceux qui le souhaitent, là où les compétences répondent aux stricts besoins à court terme de l'entreprise.

\section{Liberté de choisir, pouvoir d'agir et potentiel de développement}

Les trois dimensions constitutives des capacités amènent à formuler la question de la formation conjointement en termes de choix, de pouvoir de réalisation et de potentiel de développement. C'est à travers ces trois dimensions que nous évaluerons la contribution de la formation continue à la liberté professionnelle et à la sécurisation des parcours. Elles mettent en exergue la part humaine du développement économique et appellent à concilier une conception de la personne comme fin en soi avec la conception, consubstantielle à l'activité économique capitaliste, de la personne comme moyen.

\section{La capacité de choisir}

La capacité de choisir saisit la formation sous l'angle de la liberté de choisir ; elle soulève la question de l'étendue des possibles et de leur accessibilité. Or, même en des matières où le droit du travail accorde une liberté formelle au salarié, cette dernière lui est souvent inaccessible en pratique. Cette difficulté se pose pour les réformes récentes du plan de formation, mais également pour le CIF qui doit permettre à « tout travailleur, tout au long de sa vie professionnelle, de suivre à son initiative des actions de formation indépendamment du plan de formation de l'entreprise ». Dans son principe, ce droit peut être considéré comme capacitant. Il vise en effet à donner accès, à l'instigation du salarié, à un niveau supérieur de qualification, à un changement d'activité ou de profession, indépendamment de la politique de l'entreprise. Des enquêtes récentes montrent cependant une grande disparité des mises en œuvre régionales du CIF. Elle révèlent aussi une nette tendance à détourner ce droit de ces objectifs initiaux, afin de le mettre au service des 
besoins des entreprises et de leurs plans de formation, au détriment des projets individuels de développement professionnel (Podevin et al., 2008). Bigtrucks détonne sur ce point en encourageant les projets individuels de formation qualifiante et en offrant de réelles possibilités à ses salariés de s'y engager. L'entreprise finance rarement des formations diplômantes longues, mais le service des ressources humaines apporte son soutien à la formulation de projets personnels d'évolution ou de reconversion orientés vers des sources externes de financement du type congé individuel de formation. Ainsi parmi la trentaine d'ouvriers rencontrés, trois ont monté un dossier CIF. Ils en soulignent la complexité et l'importance de l'appui du service des ressources humaines, là où d'autres entreprises laissent les salariés se tourner vers les délégués du personnel ou se débrouiller seuls. Cet accompagnement permet de compenser l'inégal accès à l'information et aux compétences nécessaires au montage du dossier et, accessoirement, d'accroître les chances d'aboutissement des demandes. Sans cet appui, certains opérateurs n'auraient pas été en mesure d'accéder à un congé formation, alors que, formellement, il s'agit d'un droit de tirage accessible à tous. Cet exemple illustre l'importance de l'accompagnement des parcours pour l'ouverture de possibles et l'extension de la latitude de choix, tout particulièrement pour les personnes les moins pourvues en qualifications et ressources sociales.

\section{Le pouvoir de réalisation}

Le pouvoir de réalisation thématise la formation sous l'angle des accomplissements. Il est relatif aux moyens d'agir - individuels et collectifs - et aux conditions de leur mise en œuvre dans une situation donnée. La liberté comme pouvoir d'être et de faire implique qu'une personne soit à même de convertir les opportunités et les ressources disponibles en accomplissements de valeur - par exemple la conversion d'un projet personnel de formation en une formation qualifiante ; cela suppose que les différents éléments, personnels et environnementaux, qui façonnent sa capacité d'agir, se combinent adéquatement. Les facteurs de conversion contribuent à cette mise en adéquation. Les parcours professionnels montrent leur variété. Un seul suffit rarement à fonder la capacité d'une personne, qui résulte plutôt de l'interaction entre différents facteurs. Prenons le cas d'Olivier. Agé de 30 ans et titulaire d'un CAP (certificat d'aptitude professionnelle) de tourneur, Olivier est entré chez Bigtrucks en tant que magasinier, il y a dix ans. Il a depuis gravi tous les échelons du grade d'opérateur. Son « idéal » est de devenir contremaître, mais sa détermination, l'investissement au travail et la compétence reconnue dont il fait preuve n'y suffisent pas. Dans une entreprise où les postes de contremaître sont convoités et rares, la qualification joue un rôle de filtre, avec le Bac ou son équivalent comme minimum requis pour sortir de la condition ouvrière. Encouragé par son supérieur hiérarchique à reprendre des études, Olivier quitte l'usine pendant huit mois pour suivre une formation de technicien de stockage et passer l'équivalent d'un Bac dans le cadre d'un CIF. La réalisation du projet d'Olivier est rendue possible par l'interaction de différents facteurs de conversion : des facteurs d'ordre institutionnel - l'existence du CIF qui permet d'accéder à une formation qualifiante avec un maintien des revenus; d'ordre personnel et familial - sa détermination, ses compétences et le soutien de sa compagne ; d'ordre relationnel - l'appui de son supérieur hiérarchique qui le conseille et opère comme un passeur entre Olivier et le service des ressources humaines de l'entreprise ; enfin d'ordre organisationnel - le service des ressources humaines qui est à l'écoute de sa demande, délivre le mode d'emploi des dispositifs adéquats, l'aide à construire son dossier et appuie son projet. L'exemple d'Olivier montre que les seuls dispositifs légaux et conventionnels (facteurs d'ordre institutionnels) ne suffisent pas à fonder la capacité à se former s'ils ne se combinent pas avec d'autres facteurs qui façonnent le pouvoir d'agir dans l'entreprise ${ }^{35}$.

\section{Le potentiel de développement}

Le potentiel de développement saisit la formation à travers les valeurs qui la sous-tendent et les objectifs vers lesquels elle est orientée. Dans le cas d'Olivier, ce potentiel se mesure en termes d'impact de la formation sur le développement professionnel et la carrière. Au moment de l'entretien, Olivier résume avec lucidité l'objectif de sa formation : «Je ne fais

35 Pour une liste, non exhaustive, de facteurs de conversion, cf. Zimmermann (2010). 
pas la formation pour un autre poste qui m'attend au bout, c'est plus pour avoir des capacités à postuler sur des postes ». La formation n'enclenche pas automatiquement la promotion; elle donne les atouts pour concourir aux postes de catégorie supérieure, lorsqu'il s'en libère. Telle est la dimension capacitante de la formation dans le cas d'Olivier.

Son parcours est représentatif des parcours de développement professionnel ouvrier les plus réussis dans l'usine : des parcours de jeunes, recrutés pour leur premier emploi, qui ont adhéré à l'« esprit Bigtrucks » instillé par le management participatif, et qui ont su en tirer le meilleur parti et le perpétuer. De ce point de vue, Bigtrucks dément empiriquement le discours convenu sur l'impossible mise au travail des jeunes, leur manque de sérieux et de persévérance, souvent entendu au cours de l'enquête. La motivation et la capacité à travailler ne sont pas imputables à la seule personne du travailleur; elles se forgent dans la relation aux autres et à l'organisation du travail. Le respect et la confiance, l'ouverture de perspectives d'avenir, à travers la formation notamment, aussi limitées soientelles, en sont des éléments décisifs.

\section{Le développement professionnel, gage de liberté et de sécurité}

Le développement professionnel peut être considéré comme une visée et une résultante de la logique de capacités dans l'entreprise. Lié à l'employabilité, il est source de sécurité et d'expansion des libertés réelles dont disposent les personnes; les capacités en sont à la fois le moyen et la finalité. Le développement professionnel ne signifie alors pas forcément faire carrière ou prendre des responsabilités ; il n'est pas réductible au développement de connaissances et de savoir-faire. Il intègre des considérations d'épanouissement personnel et d'équilibre entre vie professionnelle et vie privée. Il appelle la possibilité pour le salarié de poser des choix, de construire un projet professionnel en accord non seulement avec les besoins de l'organisation, mais encore avec la finalité qu'il attribue lui-même au travail. Cela suppose l'existence d'espaces de discussion qui permettent de faire valoir ces choix, de même que l'existence d'opportunités et de moyens (de formation notamment) pour réaliser l'option choisie. Si l'entreprise n'offre pas ces conditions élémentaires, le salarié seul ne peut les créer ; c'est pourquoi le développement professionnel est une co-production entre le salarié et l'entreprise. Afin de ne pas être l'apanage des plus débrouillards ou des mieux placés dans l'organisation, il requiert, à des fins de justice sociale, des supports collectifs, d'ordre institutionnel et organisationnel, à même d'étayer l'égale liberté de se développer professionnellement. Or les accords collectifs et les politiques de ressources humaines des entreprises n'y suffisent pas. Ils n'ont que peu d'impact s'ils ne sont pas relayés par un management et une organisation du travail favorables aux capacités.

L'enquête a permis d'identifier quatre capacités constitutives du développement professionnel : la capacité à exercer un emploi et un travail de qualité, la capacité à trouver un équilibre entre vie professionnelle et vie privée, la capacité à se former, enfin la capacité à s'exprimer et à se faire entendre (Zimmermann, 2010). Un emploi de qualité se définit à travers les modalités de recrutement, le type de contrat, les horaires, le salaire, l'accès à la formation, autant de caractéristiques constitutives de la qualité de la relation salariale. Aussi décisive qu'elle soit, cette dernière ne suffit cependant pas à asseoir le développement professionnel ; la qualité du travail, saisie à travers ses conditions concrètes de réalisation, s'avère tout aussi déterminante. La qualité du travail n'est pas redevable de la seule performance $\mathrm{du}$ travailleur, elle est aussi tributaire de l'environnement et des conditions de réalisation du travail. Elle peut être considérée comme un bien partagé coproduit par l'entreprise et les salariés : un bien collectif vers lequel convergent les intérêts des différentes parties. Source de développement économique pour l'entreprise, la qualité du travail participe à la sécurisation des parcours des salariés, au même titre que la qualité de l'emploi.

La capacité à équilibrer vie professionnelle et privée concerne la possibilité de concilier le travail avec des choix de vie extra-professionnels. Elle inclut la liberté de choisir de ne pas faire carrière si le salarié considère que cela signifierait plus de responsabilité, plus de stress, plus de temps passé au travail et par conséquent moins de disponibilité pour la vie de famille ou ses activités extra-professionnelles. Entendu en termes de capacités, le développement professionnel intègre donc le respect des arbitrages et des hiérarchies 
que les personnes sont amenées à établir entre leurs différents engagements et qui ne placent pas nécessairement la carrière en pôle position. Venons-en maintenant à la capacité à se former ${ }^{36}$ et la capacité à s'exprimer et à se faire entendre, sur lesquelles se centre plus directement notre propos.

\section{La formation comme "bien en soi " et ouverture de possibles}

La formation peut être envisagée de deux manières : d'une part, comme «bien en soi », au sens d'objectif jugé digne d'être poursuivi en tant que tel (Dodier, 2003, p. 19) ; d'autre part, comme ouverture de possibles, et potentiel de développement. Au titre de bien en soi comptent les réalisations en matière de formation. Elles se mesurent en termes de volume, de distribution et de qualité. Au titre d'ouverture de possibles, on compte les réalisations qu'autorise la formation, notamment en matière de développement professionnel. Ancrée dans le présent en tant que bien en soi, la formation est orientée vers l'avenir à travers les possibles qu'elle ouvre.

L'évaluation de la politique de formation des entreprises se cantonne généralement à la mesure de la formation comme bien en soi, alors que l'ouverture des possibles et les débouchés de la formation, beaucoup plus rarement évalués, constituent une contribution décisive de la formation à la liberté professionnelle et la sécurisation des parcours. En tenir compte induit un déplacement de l'attention de la seule formation vers la capacité de développement susceptible d'en découler. Or les statistiques montrent que si, depuis 1977, le taux des formés a considérablement augmenté, les durées des formations ont en revanche drastiquement fondu et la certification s'est effondrée (Bérêt, 2009, p. 72). Pourtant, lorsque l'entreprise s'engage dans le développement professionnel de ses salariés, elle peut en tirer autant bénéfice que ces derniers.

C'est ce que suggère l'exemple de Bigtrucks, où le développement professionnel n'emprunte pas seulement la voie de la formation formelle, mais aussi de l'apprentissage en situation de travail. L'entreprise donne accès à une combinaison de différents types

\footnotetext{
${ }^{36} \mathrm{Qu}$ 'il convient de distinguer des formations « veillant au maintien de la capacité à occuper un emploi » précédemment évoquées et relevant de la responsabilité juridique de l'employeur.
}

de savoir et de savoir-faire : des savoirs spécifiques à l'entreprise, des savoirs spécifiques au secteur automobile, enfin des savoirs plus généralistes. L'acquisition des savoirs généralistes passe autant par la formation individuelle, à l'anglais par exemple, que par des dispositifs de management participatif tels que, selon le modèle toyotiste, les groupes de résolution de problèmes ou les délégations qui amènent les ouvriers à quitter la chaîne quelques heures par mois ou par semaine pour se consacrer à d'autres tâches. Les groupes de résolution de problèmes réunissent de manière $a d$ hoc des salariés de différents rangs hiérarchiques pour traiter de l'adaptation d'une portion de ligne (cluster) à un changement de cadence ou à l'assemblage d'une nouvelle série de camions, mais aussi de problèmes humains et relationnels. Les délégations installées sur la durée vont de l'approvisionnement en petit outillage ou du traitement des non-conformités au sein d'un cluster à des fonctions d'auditeur ${ }^{37}$ ou de guide pour les visiteurs. Ces différents dispositifs favorisent les apprentissages collectifs en situation, la prise d'initiative et de responsabilité. Comparativement aux autres savoirs, le propre des savoirs généralistes est leur transférabilité au-delà de l'entreprise, voire même du secteur. C'est à ce titre qu'ils contribuent, combinés à des savoirs plus spécifiques, à la capacité de développement professionnel de l'ouvrier chez Bigtrucks.

Mais le mode d'acquisition des savoirs se révèle tout aussi décisif que les savoirs eux-mêmes. L'association de sessions instituées de formation à des apprentissages sur le tas et une mutualisation, voire une élaboration collective, des savoirs dans le cadre du management participatif offrent une palette de méthodes qui se complètent. Leur caractéristique, du point de vue du développement professionnel, est de ne pas se réduire à des canaux de transmission. Elles intègrent en outre des formes de production du savoir en situation et promeuvent un rapport actif à la connaissance. Autant que la formation en tant que telle, c'est le processus d'apprentissage permanent, organisé par le management participatif, qui contribue au développement professionnel chez Bigtrucks.

\footnotetext{
${ }^{37} \mathrm{La}$ fonction d'auditeur consiste à auditer des camions prélevés de manière aléatoire à la sortie de la ligne.
} 
La formation continue, souvent mise en exergue comme moyen privilégié du développement professionnel, n'en constitue par conséquent qu'un élément parmi d'autres. Quels que soient son intérêt et sa qualité, une formation n'est pas automatiquement un facteur de développement. Encore faut-il qu'en amont le salarié ait les moyens d'orienter sa formation, c'està-dire la capacité d'exprimer ses préférences et de les faire valoir. Encore faut-il qu'une fois réalisée, cette formation débouche sur un travail de qualité dans un emploi de qualité et que ce travail se révèle compatible avec l'équilibre entre vie personnelle et professionnelle auquel aspire le salarié.

\section{Participation, capacité à s'exprimer et liberté professionnelle}

La capacité à s'exprimer et à se faire entendre est un élément décisif de la liberté professionnelle. Par delà l'accès à l'information et la participation, elle suppose que chaque salarié soit à même de faire connaître, dans la situation qui est la sienne, ce qui vaut pour lui en matière de travail et d'emploi, de formation, d'équilibre entre vie professionnelle et privée. Elle engage donc un processus de valuation, au sens d'un processus d'attribution de valeur, de détermination de ce qui vaut et de hiérarchisation (Dewey, 1988), qui étaye l'expression des préférences. Parce que la capacité d'expression implique tout à la fois une capacité de valuation et une capacité à se faire entendre, elle structure de manière transversale l'ensemble des autres capacités.

Une formation est d'autant plus sécurisante qu'elle offre au salarié une possibilité de prise sur son devenir. Et cette possibilité est d'autant plus grande que la personne peut faire valoir ses préférences. C'est là une dimension du développement et de la liberté professionnelle trop souvent négligée dans les débats sur la sécurisation des parcours. Chez Bigtrucks, « on a l'avantage de pouvoir discuter avec nos responsables sans forcément attendre l'entretien annuel » (Olivier). Ce n'est pas un hasard si les organisations qui se distinguent par une politique de développement professionnel largement accessible aux salariés, sans discrimination socioprofessionnelle, se caractérisent aussi par les possibilités de participation et d'expression qu'elles offrent en dehors du strict cadre de l'entretien annuel.
L'extension de la gamme de choix des personnes au travail, de leur capacité à aspirer et à faire valoir ce qui vaut pour elles pose la question éminemment politique de la gouvernance de l'entreprise et des formes de participation des salariés. Être un agent actif de son propre développement suppose de pouvoir participer aux décisions qui influent sur son existence, c'est-à-dire avoir une possibilité de prise non seulement technique, mais également politique et morale sur son environnement. Les organisations syndicales ont, au cours du $\mathrm{xx}^{\mathrm{e}}$ siècle, offert une telle possibilité en instaurant une médiation fondée sur la participation par délégation, appuyée sur un système électif de représentation. Le propre de cette médiation était de promouvoir une prise collective sur les destinées individuelles à l'intérieur de grandes catégories socioprofessionnelles, comme l'illustrent les grilles de classification des postes ou des fonctions par branches professionnelles. La participation sous contrôle managerial oppose à cette stratégie représentative une logique de contrôle individualisé du devenir, assise sur la participation directe et la relation de face à face entre le travailleur et son supérieur hiérarchique ; pratiques susceptibles d'ouvrir la voie aux pires abus de pouvoir si elles ne sont pas assorties d'une politique de capacités individuelles collectivement garanties.

En ouvrant un espace, même limité, de délibération sur le travail, la participation capte, chez Bigtrucks, la capacité critique et d'expression des personnes au service de l'entreprise. C'est ce qu'en retiennent les responsables en recrutement des entreprises voisines. Les ouvriers, à l'inverse, y voient la chance de pouvoir contribuer à définir, bien que sur des points circonscrits et encadrés par la direction, comment ils veulent travailler. En dépit d'un cadrage rigoureux - les décisions stratégiques ne sont jamais mises en délibération, seule leur mise en œuvre l'est -, la participation produit, chez les ouvriers Bigtrucks, un sentiment de prise sur le cours des choses, aussi bien au niveau de leur activité que de leur parcours, de même qu'un sentiment de mieux-être au travail, comparativement aux autres expériences professionnelles qu'ils ont pu connaître. Si le management participatif est un instrument de normalisation des comportements à travers la dimension morale qu'il assigne au travail - en faisant de la participation un devoir-être -, la manière dont 
il est mis en œuvre chez Bigtrucks contribue aussi au développement professionnel et à la réputation de qualité des salariés.

\section{La qualité du salarié, une autre manière d'articuler flexibilité et sécurité}

Le cas Bigtrucks est significatif de ce que produit une formation orientée vers le développement professionnel. Elle ne constitue pas nécessairement une carrière, mais ce qu'on appellera la qualité du salarié, localement reconnue et recherchée. La qualité du salarié procède d'une assignation de capacités qui n'est pas circonscrite à une activité professionnelle ou à des compétences données. Elle préjuge d'un potentiel d'apprentissage et d'une capacité d'adaptation à des situations changeantes. La capacité à se former, à développer et renouveler ses compétences en est une composante essentielle. La formation participe à la réputation de qualité du salarié Bigtrucks à double titre : comme «bien en soi », à travers la qualité des apprentissages, leur caractère transversal et leur durabilité, mais aussi et surtout comme ouverture de possibles et potentiel de développement. C'est plus largement le développement professionnel - recouvrant outre la capacité à se former, la capacité à exercer un emploi et un travail de qualité, à s'exprimer et participer, à équilibrer vie privée et vie professionnelle - qui forge la qualité du salarié Bigtrucks.

La qualité du salarié Bigtrucks jouit d'une réputation solidement ancrée auprès des autres employeurs et salariés du bassin d'emploi. Partagée par l'ensemble des membres de l'entreprise, elle met en exergue la dimension collective des capacités qui, avant d'être des attributs individuels, sont tributaires des condi- tions et des possibilités offertes par l'organisation. C'est parce que la qualité du salarié est associée au caractère capacitant de l'organisation que tous les salariés en sont a priori crédités, indépendamment de leur fonction, qualification ou classification. Recherché par les autres entreprises, mais également leurs salariés, le label Bigtrucks ouvre à ses détenteurs un espace potentiel de mobilité qui motive leur confiance en l'avenir. La différence entre des compétences dont l'individu est le seul garant et des capacités dont un collectif - en l'occurrence de travail - se fait le garant, apparaît ici déterminante en matière de sécurisation des parcours. Cela ne signifie pas que les capacités se substituent aux compétences individuelles; elles se situent à un autre niveau et offrent un cadre qui permet, en autres, l'expression et le développement de ces dernières.

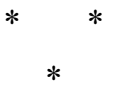

La mise en œuvre française de la flexicurity comme du lifelong learning, à travers la sécurisation des parcours professionnels, met l'accent sur des dispositifs de nature contractuelle essentiellement orientés vers une formation liée au poste et au maintien dans l'emploi, au détriment d'un réel droit individuel du salarié. $\mathrm{La}$ capacité à saisir ces dispositifs et à les transformer en facteurs de sécurisation des parcours est décisive. La capacité dessine un scénario d'articulation entre sécurité et flexibilité qui fait de la liberté professionnelle également accessible à tous son idéal normatif. La formation en est un élément important, mais n'y trouve sa pleine expression qu'envisagée sous l'angle $\mathrm{du}$ développement professionnel qu'elle autorise, au-delà des seuls droits formels qui l'instituent.

\section{Bibliographie}

Béret P. (2009), «Formation continue, salaires et transformation des marchés internes », Travail et Emploi, ${ }^{\circ} 117$, pp. 67-80.
Commission européenne (2001), Réaliser un espace européen de l'éducation et de formation tout au long de la vie, $\operatorname{COM(2001)~} 678$ final. 
Commission européenne (2007), Vers des principes communs de flexicurité : des emplois plus nombreux et de meilleure qualité en combinant flexibilité et sécurité, Bruxelles, Com 359 final.

Corteel D., Zimmermann B. (2007), «Capacités et développement professionnel », Formation Emploi, $\mathrm{n}^{\circ} 98$, pp. 25-39.

Deakin S. et Supiot A. (2009), Capacitas, Oxford, Hart Publishing.

De Munck J., Zimmermann B. (2008), La liberté au prisme des capacités. Amartya Sen au-delà du libéralisme, Paris, EHESS.

Dewey J. (1988 [1939]), Theory of valuation in The later Works, 1925-1953, Carbondale and Edwardsville, Southern Illinois University Press.

Dodier N. (2003), Leçons politiques de l'épidémie du sida, Paris, Éditions de l'EHESS.

Favennec-Hery F. (2007), «La sécurisation des parcours professionnels, sésame de toutes les réformes ? », Droit social, n 663, pp. 1105-1109.

Gaudu F. (2008), «De la flexicurité à la sécurité sociale professionnelle: l'emploi entre mobilité et stabilité », Formation Emploi, n ${ }^{\circ}$ 101, pp. 71-88.

Gutmann D. (2003), « Droits subjectifs », in Alland D., Rials S. (dir.), Dictionnaire de la culture juridique, Puf / Lamy, p. 530.

Hasan A. (1997), «La formation tout au long de la vie ", Revue internationale d'éducation, $\mathrm{n}^{\circ} 16$, décembre.

Lambert M., Vero J., Corteel D. et Zimmermann B. (2009), « Capability for lifelong learning in French companies ", in Bartelheimer P., Moncel N. (dir.), Sen-sitising life course research? Exploring Amartya Sen's capability concept in comparative research on individual working lives, Cereq, Net.doc, $\mathrm{n}^{\circ} 50$, pp. 115-157.

Luttringer J.-M. et Willems J.-P. (2008), « Sécurisation des parcours professionnels : "Réflexions juridiques à propos d'un oxymore" ", AEF Document n ${ }^{\circ} 1439$.
Maggi-Germain N. et Caillaud P. (2007), «Vers un droit personnel à la formation », Droit Social, $\mathrm{n}^{\circ} 659$, pp. 574-591.

Maggi-Germain N. (2008), « Formation professionnelle continue et sécurisation des parcours professionnels », Semaine sociale Lamy Supplément, $\mathrm{n}^{\circ} 1348$, pp. 21-30.

Pochet P. (2004), « La MOC et la protection sociale : des développements ambigus », in Dehousse R., eds, L'Europe sans Bruxelles. Une analyse de la méthode ouverte de coordination, Paris, L'Harmattan, pp. 99-130.

Podevin G., Ghaffari S., Caillaud P., Dubard C. (2008), Le congé individuel de formation : un droit national, des réalités territoriales, Rennes, PU Rennes.

Sen A. (2003), « La liberté individuelle : une responsabilité sociale ", in L'économie est une science morale, Paris, La Découverte.

Subramanian D., Zimmermann B. (à paraître en 2011), What are the drivers for Companies'Training Policies?

Supiot A. (1997), « Du bon usage des lois en matière d'emploi », Droit Social, mars, pp. 229-242.

Supiot A. (1999), Au-delà de l'emplo ; transformations du travail et devenir du droit du travail en Europe, Rapport à la Commission européenne, Paris, Flammarion.

Supiot A. (2002), Critique du droit du travail, Paris, PUF.

Vatinet R. (2007), « Nouveaux cadres juridiques pour les parcours professionnels diversifiés », La Semaine Juridique édition sociale, $\mathrm{n}^{\circ}$ 9, pp. 1131-1141.

Zimmermann B. (à paraître en 2011), Ce que travailler veut dire. Une sociologie des capacités et des parcours professionnels, Paris, Economica. 


\section{Résumé}

\section{Sécurisation des parcours et liberté professionnelle : de la «flexicurité » aux capacités \\ Pascal Caillaud et Bénédicte Zimmerman}

À partir d'une double approche juridique et sociologique, cet article analyse, dans un premier temps, la transposition dans le droit français des concepts européens de Flexicurity et de lifelong learning. II confronte ensuite les droits formels à leur mise en œuvre par les entreprises, à partir de deux enquêtes qualitatives sur le développement professionnel des salariés, menées en 2005 et 2009. L'approche par les capacités de A. Sen est mobilisée pour définir la formation conjointement en termes de choix, de potentiel de développement et de pouvoir de réalisation. Une telle définition ouvre sur la notion de développement professionnel, à travers laquelle les auteurs identifient une autre manière d'articuler flexibilité et sécurité, accordant aux conditions de réalisation du travail un rôle tout aussi décisif qu'aux conditions d'emploi.

\section{Mots clés}

Approche par les capabilités, sécurisation des parcours professionnels, cheminements professionnels, droit individuel à la formation

Journal of Economic Literature: M 54, J 62 